\title{
MOTIVACIONES, NECESIDADES E IMPORTANCIA ATRIBUIDA A LA FORMACIÓN CONTINUA EN EL DESARROLLO DE HABILIDADES PROFESIONALES DEL PROFESORADO EN LA ENSEÑANZA DE ENFERMERÍA
}

\section{Motivations, needs and importance attributed to continous training in the development of professional skills of teaching staff in nursing education}

\author{
Rogério Manuel Ferrinho FerreIRA*, Sixto Cubo DelgadO*** \\ y Vito José de Jesus Carioca****k \\ *Instituto Politécnico de Beja (Portugal). Correo-e: ferrinho.ferreira@ipbeja.pt \\ **Universidad de Extremadura.Correo-e: sixto@unex.es \\ ***Instituto Politécnico de Beja (Portugal). Correo-e: vcarioca@ipbeja.pt
}

Recibido: 26-6-2016; Aceptado: 22-3-2017; Publicado: 30-11-2017

Ref. Bibl. ROGÉRIO MANUEL FERRINHO FERREIRA, SIXTO CUBO DELGADO Y VITO JOSÉ DE JESUS CARIOCA. Motivaciones, necesidades e importancia atribuida a la formación continua en el desarrollo de habilidades profesionales del profesorado en la enseñanza de enfermería. Enseñanza \& Teaching, 35, 2-2017, 77-96.

RESUMEN: Este estudio tuvo como principal objetivo investigar la importancia que los profesores de enseñanza de enfermería de instituciones públicas de Educación superior de Portugal atribuyen a la formación continua en el desarrollo de las capacidades profesionales, teniendo en consideración las principales motivaciones de los profesores para participar en acciones de formación y/o de desarrollo continuo, así como sus necesidades de formación continua.

En este estudio, participaron 157 profesores, seleccionados de forma aleatoria. Los participantes respondieron a un cuestionario que fue evaluado por expertos 
ROGÉRIO MANUEL FERRINHO FERREIRA, SIXTO CUBO DELGADO Y VITO JOSÉ DE JESUS CARIOCA MOTIVACIONES, NECESIDADES E IMPORTANCIA ATRIBUIDA A LA FORMACIÓN CONTINUA EN EL DESARROLLO DE HABILIDADES PROFESIONALES DEL PROFESORADO EN LA ENSEÑANZA DE ENFERMERÍA

alcanzando un índice de validez del contenido de 0,94. La fiabilidad de los instrumentos de medida se obtuvo a través del coeficiente Alfa de Cronbach, en la variable de «Motivaciones para la participación en acciones de formación y/o de desarrollo continuo» el coeficiente obtenido fue de 0,811 ; en la variable de «Necesidades actuales de formación continua» de 0,876 , y, por último, en la variable de "Importancia atribuida a la formación continua en el desarrollo de habilidades profesionales del profesor de Educación Superior» de 0,876. Por lo que el estudio revela una elevada consistencia interna.

Los resultados obtenidos evidencian que la muestra seleccionada de profesores atribuyen importancia a la formación continua en el desarrollo de habilidades profesionales; sobresaliendo en las categorías de «Preparar los contenidos de las unidades temáticas», "Garantizar la orientación tutorial», «Relacionarse con los estudiantes», «Evaluar", "Utilizar las tecnologías de información y comunicación» y "Planificar el proceso de enseñanza-aprendizaje». En las motivaciones que provocan la participación de los profesores en acciones de formación y/o de desarrollo continuo se destacan las categorías «Motivos Prácticos» y "Motivos Emancipadores». Por último, en relación a las necesidades actuales de formación continua sobresalen las categorías relacionadas con «Investigar», «Utilizar las tecnologías de información y comunicación» y «Evaluar».

Palabras clave: formación continua; motivaciones; habilidades profesionales; Enseñanza superior y enfermería.

SUMMARY: This study, of descriptive nature, had as its main purpose to investigate the importance that nursing education professors, in public higher education institutions, in Portugal, assign to continuous training on the development of professional skills, taking into account the professors main motivations to participate in training activities and/or continued development and their needs of further continuous training.

157 randomly selected professors, who completed a questionnaire, participated on it. In its assessment by judges/experts, a 0,94 content validation index was achieved. Regarding the reliability of the measuring tool in the "Motivations for engagement in training actions and/or continuous professional development» category, there was a Cronbach's Alpha of 0,811 , in the "Continuous training present needs" category, a Cronbach's Alpha of 0,876 was presented and finally, in the dimension «Emphasis placed on the continuous training in the development of higher education professor skills» a Cronbach's Alpha of 0,978 was shown, compliant with a high internal consistency.

The achieved results allow us to imply that these professors give credit to the continuous training on the development of professional skills, with emphasis on the skills of "Preparing the content of the curriculum units", "Assure tutorial orientation", "Communicate with the students", "Evaluate", "Use of information and communication technologies" and "Planning the teaching-learning process». The "Practical Reasons» and the "Emancipatory Reasons", stand out in the motivations that move professors to participate in training and/or continuous development actions and, with regard to the current needs of continuous training, the ones related to investigating, use of information and communication technologies and evaluation stand out. 
ROGÉRIO MANUEL FERRINHO FERREIRA, SIXTO CUBO DELGADO Y VITO JOSÉ DE JESUS CARIOCA

MOTIVACIONES, NECESIDADES E IMPORTANCIA ATRIBUIDA A LA FORMACIÓN CONTINUA EN EL DESARROLLO DE HABILIDADES PROFESIONALES DEL PROFESORADO EN LA ENSEÑANZA DE ENFERMERÍA

Key words: Continuous training; Motivations; Skills; Higher Education and Nursing.

\section{INTRODUCCIÓN}

Es necesario apostar por la formación continua como repuesta a los desafíos de una sociedad en constante transformación, así como para los nuevos desafíos de la educación. La formación continua se torna condición esencial y parte integrante del proceso de desarrollo de los profesores (Flores, Veiga Simão, Rajala y Tornberg, 2009).

El profesor es un profesional que asume múltiples roles, que envuelven un conjunto de capacidades científicas y pedagógicas (Cadório y Veiga Simão, 2013; Santos, 2013), que le permiten configurar sus prácticas docentes, tanto en función de los diferentes contextos donde se desarrolla el aprendizaje, como de la particularidad de las diferentes situaciones. Le permite, también, garantizar su función como facilitador del aprendizaje de los estudiantes (Almeida, 2012; Cunha, 2010; Fernandes, 2010; García Sanz y Maquilón Sánchez, 2010; Zabalza, 2012). El profesor es un aprendiz durante toda la vida (Cadório y Veiga Simão, 2013), por lo que su desempeño como profesional de la enseñanza no se puede disociar de su propio aprendizaje. Por tanto, se puede afirmar que la profesión de profesor se determina por la formación y por los conocimientos profesionales inherentes a su práctica profesional (Almeida, 2012; Esteves, 2009; Marcelo, 2009; Mesquita, 2010).

La formación continua abarca una gama de actividades de las instituciones después de la formación inicial y que tienen como objetivo la mejoría profesional del profesor, para beneficio de los alumnos y de la escuela (Correia, 2012). Se trata de un concepto que aparece asociado al desarrollo profesional, a través del aprendizaje provocado por estos procesos (Cadório y Veiga Simão, 2013; Correia, 2012; Flores et al., 2009). La formación continua, desde una perspectiva de desarrollo profesional es vista como un proceso individual y/o colectivo, permanente y continuo, que contribuye al desarrollo de las capacidades profesionales del profesor (Ferreira, Pereira y Xavier, 2012).

La formación continua surge asociada al concepto de desarrollo profesional, a través del aprendizaje que se encuentra implícito en el mismo (Cadório y Veiga Simão, 2013; Correia y Flores, 2009; Flores et al., 2009; González Calvo y Barba, 2014). Puede ser vista como parte o componente del proceso de desarrollo profesional de los profesores (Eirín Nemiña, García Ruso y Montero Mesa, 2009) o puede tener como consecuencia el desarrollo profesional del profesor (Correia, 2012).

Desde la perspectiva del desarrollo profesional, la formación continua se debe afrontar como un proceso individual, permanente y continuo, que engloba el crecimiento personal a lo largo de la vida, así como el desenvolvimiento y actualización de la actividad profesional. Por lo que la formación continua forma parte integrante de las prácticas sociales y actividades cotidianas (Ferreira et al., 2012). Es, también, un proceso colectivo que debe ser concretizado en la escuela, a través de diferentes 
ROGÉRIO MANUEL FERRINHO FERREIRA, SIXTO CUBO DELGADO Y VITO JOSÉ DE JESUS CARIOCA MOTIVACIONES, NECESIDADES E IMPORTANCIA ATRIBUIDA A LA FORMACIÓN CONTINUA EN EL DESARROLLO DE HABILIDADES PROFESIONALES DEL PROFESORADO EN LA ENSEÑANZA DE ENFERMERÍA

experiencias de índole formal e informal y que contribuyen al desarrollo de las habilidades profesionales del profesor (Ferreira et al., 2012; Marcelo, 2009; Murta, 2013).

El aprendizaje puede existir en diferentes contextos asociados a la formación y a los procesos formativos. Correia (2012), Flores et al. (2009) y Eirín Nemiña et al. (2009) hacen referencia al método de instrucción directa (conferencias, cursos, seminarios y workshops, etc.); al aprendizaje en la escuela (colaboración entre los docentes, críticas constructivas, procesos de evaluación, investigación-acción, evaluación de los trabajos, trabajo en grupo sobre las mismas funciones), y al aprendizaje realizado fuera de la escuela, a través de centros de desarrollo profesional, de redes temáticas, de grupos informales, entre otros. En todos estos contextos, los cuales no deben excluir el aula, el profesor construye su propio aprendizaje, que lo capacita para el cumplimiento de sus funciones educativas en múltiples contextos de intervención docente (González Calvo y Barba, 2014).

Es reconocida la importancia del desarrollo profesional y, dentro de este, de la formación continua, en el perfeccionamiento profesional del profesor y en el desenvolvimiento organizacional de la escuela. Se trata, por tanto, de un mecanismo de crucial importancia en la afirmación del docente como profesional. Esta afirmación es realizada por medio del aprendizaje implícito y de la adquisición de conocimientos y competencias, que permiten al profesor aprender, intervenir y transformar la práctica dentro de un contexto muy complejo (Almeida, 2012; Cadório y Veiga Simão, 2013; Correia, 2012; Correia y Flores, 2009; Eirín Nemiña et al., 2009; Esteves, 2009; Ferreira et al., 2012; Flores et al., 2009; Forte, 2009; Lee, 2011; Marcelo, 2009; Murta, 2013).

Las competencias son un concepto que engloba el conocimiento, el saber hacer y el saber ser/estar (Esteves, 2009; Jooste y Jasper, 2010; Zabalza, 2010). Estos tres ítems anteriores deben ser conjugados en la práctica docente, ya que se encuentran implícitos en varias ocupaciones o actividades que, a su vez, se deben conjugar en la práctica educativa y en los diferentes contextos que forman la actividad del profesor (Esteves, 2009; Mas, 2016; Mestrinho, 2012; Ricardo, 2010; Veiga Simão, Flores, Morgado, Forte y Almeida, 2009).

La formación continua contribuye a la mejoría de las competencias profesionales del profesor. Engloba el aprendizaje, las alteraciones de las competencias profesionales y el desarrollo del profesor (Veiga Simão et al., 2009).

El profesor interviene en cada situación educativa mediante un conjunto de competencias (Mas, 2016). Ante el alcance y la lógica del paradigma de aprendizaje y a raíz de la creación del Espacio Europeo de Educación Superior, adquirimos como referencia el conjunto de competencias para el profesor de Educación Superior definido por Zabalza (2010). Según este autor este conjunto de competencias para el profesor de Educación Superior comprende los siguientes ítems: planificar el proceso de enseñanza-aprendizaje, preparar los contenidos de las unidades temáticas, dar clase de forma comprensible y bien organizada, utilizar los medios de información y comunicación, definir la metodología y/u organizar 
ROGÉRIO MANUEL FERRINHO FERREIRA, SIXTO CUBO DELGADO Y VITO JOSÉ DE JESUS CARIOCA

MOTIVACIONES, NECESIDADES E IMPORTANCIA ATRIBUIDA A LA FORMACIÓN CONTINUA EN EL DESARROLLO DE HABILIDADES PROFESIONALES DEL PROFESORADO EN LA ENSEÑANZA DE ENFERMERÍA

las actividades, relacionarse con los estudiantes, garantizar las tutorías, evaluar, investigar e identificarse con la institución.

Las motivaciones que llevan a la formación continua son la clave para el desenvolvimiento de procesos de formación y desarrollo continuo. Este enfoque comporta la valorización de motivaciones instrumentales, motivaciones prácticas, motivaciones emancipadoras, motivaciones políticas y motivaciones pedagógicas, con algunas adaptaciones en relación con los estudios realizados por Correia (2012) y Forte (2009).

Los motivos instrumentales son aquellos que están relacionados con la progresión en la carrera, las oportunidades y las habilidades profesionales. Los motivos prácticos implican la resolución de problemas o requisitos relacionados con la propia enseñanza, mientras que los motivos emancipadores están asociados al propio desenvolvimiento del profesor. Las motivaciones políticas están relacionadas con la implementación de políticas educativas nacionales y/o locales y, por último, tenemos las motivaciones pedagógicas que surgen asociadas al aumento de objetivos que, a su vez, están relacionados con la necesidad de mayor eficacia de la enseñanza.

Por otro lado, las necesidades de formación son carencias de conocimientos, aspiraciones, dificultades, deseos, preocupaciones o expectativas del profesor en relación con sus actividades diarias (Januário, Ferro, Anacleto y Henrique, 2009), las necesidades de formación deben ser efectuadas, para así mejorar el desarrollo de las habilidades profesionales (Veiga Simão et al., 2009; Zabalza, 2010, 2012).

Fue a partir de este conjunto de reflexiones, acerca del profesor y de los procesos de formación, que decidimos realizar esta investigación, que tiene como eje central investigar la importancia que los profesores de Educación Superior atribuyen a la formación continua en el desarrollo de las habilidades profesionales. Este estudio se afirma en la convicción de que los profesores participan en diferentes cursos de formación de desarrollo profesional continuo movidos por necesidades específicas de formación, así como por diferentes motivaciones procurando el desarrollo de sus habilidades profesionales.

\section{MÉTODO}

En esta investigación fue usada una metodología de carácter descriptivo, cuyo propósito es analizar la importancia atribuida a la formación continua en el desarrollo de las habilidades profesionales en el profesor de Educación Superior, para ello contamos con la colaboración de profesores titulares de instituciones públicas portuguesas del área de enfermería.

Destaca el hecho de que este estudio obtuvo el dictamen positivo de dos instituciones de Educación Superior, indicando, pues, que cumple con los requisitos éticos en relación a mantener el anonimato y a la independencia de los participantes, por lo que reúne interés científico para su desarrollo. 
ROGÉRIO MANUEL FERRINHO FERREIRA, SIXTO CUBO DELGADO Y VITO JOSÉ DE JESUS CARIOCA MOTIVACIONES, NECESIDADES E IMPORTANCIA ATRIBUIDA A LA FORMACIÓN CONTINUA EN EL DESARROLLO DE HABILIDADES PROFESIONALES DEL PROFESORADO EN LA ENSEÑANZA DE ENFERMERÍA

\subsection{Objetivos de investigación}

Definimos los siguientes objetivos para la realización de este estudio:

- Identificar las principales motivaciones de los profesores para participar en acciones de formación y/o de desarrollo continuo;

- Identificar las necesidades de formación continua de los profesores, procurando mejorar su desempeño profesional;

- Identificar la importancia atribuida a la formación continua en el desarrollo de las habilidades del profesor.

\subsection{Participantes}

Para la realización de esta investigación se seleccionó una población objetivo constituida por 597 profesores titulares de instituciones públicas de Educación Superior de Portugal, del área científica de Enfermería. De esta población se seleccionaron de forma aleatoria 60 profesores para participar en el estudio piloto y el resto de la población, o sea 537, participaron en el estudio.

Para la aplicación del cuestionario, se seleccionó una muestra aleatoria simple de 361 profesores, de los cuales 157 respondieron al mismo.

La muestra está constituida en un $80,3 \%$ por profesores de sexo femenino y $19,7 \%$ de sexo masculino. El intervalo de edad predominante son los docentes con edades comprendidas entre los 41 a los 50 años, que representan el 51\% de la muestra; el intervalo de edad está entre los 51 a los 60 años. A destacar que el $63,1 \%$ de los profesores titulares de la muestra poseen un máster y el otro 33,1\% de la misma son doctores.

Esta muestra está formada por profesores titulares del área científica de Enfermería y, por lo tanto, estudiaron todos Enfermería como formación inicial, siendo que el 43,9\% cursaron estudios en la área científica de Enfermería y el 11,5\% en el área de Salud. El promedio de experiencia docente de estos profesores es de 17,63 años, con una desviación estándar de 8,24 años. Se constata que predominan los profesores titulares con un tiempo de servicio de 10 a 14 años, representando el $33,1 \%$; por otro lado, los profesores con un tiempo de servicio que oscila entre los 20 a los 24 años representan un 22,3\%. En relación a la categoría profesional constatamos que el $75,2 \%$ son profesores adjuntos y el otro $24,8 \%$ son profesores coordinadores. Verificamos, también, que el 59,9 de estos profesores dan clase en cursos de $2 .^{\circ}$ Ciclo, en cuanto el restante $30,6 \%$ dan clase en cursos de $1 .{ }^{\text {er }}$ Ciclo.

\subsection{Instrumentos}

El estudio se ha desarrollado a través de un cuestionario del que ofrecemos a continuación datos psicométricos. 
ROGÉRIO MANUEL FERRINHO FERREIRA, SIXTO CUBO DELGADO Y VITO JOSÉ DE JESUS CARIOCA

MOTIVACIONES, NECESIDADES E IMPORTANCIA ATRIBUIDA A LA FORMACIÓN CONTINUA EN EL DESARROLLO DE HABILIDADES PROFESIONALES DEL PROFESORADO EN LA ENSEÑANZA DE ENFERMERÍA

\subsubsection{Validez y fiabilidad}

Una vez construido el cuestionario, fue sometido a la apreciación de expertos, para evaluar tanto las dimensiones como los ítems que las componen (Fortin, Côté y Filion, 2009). Los expertos evaluaron la validez de contenido de cada ítem y de la estructura, utilizando una escala ordinal compuesta por 4 puntos: 1) no es relevante; 2) poco relevante; 3) pertinente; 4) muy relevante. Fue creado un índice de validez del contenido, que corresponde a la proporción de los enunciados que los expertos otorgan de una puntuación de 3 y 4 y el número total de enunciados (Fortin et al., 2009) y que se expresa en la siguiente fórmula:

$$
\operatorname{IVC}=\frac{\text { Número de enunciados con puntuación de } 3 \text { y } 4}{\text { Número total de enunciados }}
$$

De esta evaluación se obtuvo un índice de validez del contenido (IVc) de 0,94.

Fue posible evaluar la fiabilidad del instrumento de medida, mediante la realización de un estudio piloto previo, que incluyó 49 profesores titulares de Instituciones públicas de Portugal del área científica de Enfermería. Los resultados obtenidos ofrecen garantía en término de consistencia interna, como se puede verificar a través del Cuadro 1.

CUADRO 1

Fiabilidad del instrumento de medida

\begin{tabular}{|l|l|c|c|}
\hline Dimensión & \multicolumn{1}{|c|}{ CATEGorías } & N. o DE ÍTEMS & $\begin{array}{c}\text { ALFA DE } \\
\text { CRONBACH }\end{array}$ \\
\hline Formación continua & $\begin{array}{l}\text { Motivaciones para el des- } \\
\text { envolvimiento de acciones } \\
\text { de formación y/o desarrollo } \\
\text { profesional continuo }\end{array}$ & 16 & 0,811 \\
\cline { 2 - 4 } & $\begin{array}{l}\text { Necesidades actuales de } \\
\text { formación continua }\end{array}$ & 10 & 0,876 \\
\hline $\begin{array}{l}\text { Importancia atribuida a la } \\
\text { formación continua en el } \\
\text { desarrollo de competencias } \\
\text { del profesor de Educación } \\
\text { Superior }\end{array}$ & 68 & 0,978 \\
\hline
\end{tabular}




\section{4}

ROGÉRIO MANUEL FERRINHO FERREIRA, SIXTO CUBO DELGADO Y VITO JOSÉ DE JESUS CARIOCA MOTIVACIONES, NECESIDADES E IMPORTANCIA ATRIBUIDA A LA FORMACIÓN CONTINUA EN EL DESARROLLO DE HABILIDADES PROFESIONALES DEL PROFESORADO EN LA ENSEÑANZA DE ENFERMERÍA

\section{RESUltados}

Los resultados que presentamos representan parte de un estudio más amplio sobre la importancia atribuida por los profesores de Enfermería a la formación continua en el desarrollo de sus habilidades profesionales.

\subsection{Las motivaciones para la formación continua}

En relación con las motivaciones de los profesores para realizar formación continua, verificamos que la media de los ítems de esta categoría fue de 3,848, con una desviación típica de 0,570.

Entre las motivaciones destacan las subcategorías «Motivos Prácticos» y "Motivos Emancipadores", con medias superiores a la media total de los ítems, concretamente 4,123 y 4,160. Las motivaciones menos valorizadas fueron las Motivaciones Instrumentales $(3,344)$ y las Motivaciones Políticas $(3,397)$, como se puede constatar a través del Cuadro 2.

\section{CUADRO 2}

Medias, desviación típica y coeficientes de variación de los ítems y subcategorías en la categoría motivaciones para la formación continua

\begin{tabular}{|c|c|c|c|}
\hline SUBCATEGORÍAS & ÍTEMS & $\begin{array}{c}\text { MEDIA } \\
\text { DE LOS } \\
\text { ÍTEMS }\end{array}$ & $\begin{array}{l}\text { MEDIA DE LOS } \\
\text { ÍTEMS DE LA } \\
\text { SUBCATEGORÍA }\end{array}$ \\
\hline \multirow{3}{*}{$\begin{array}{l}\text { Motivos } \\
\text { Instrumentales }\end{array}$} & 1- Ascender en la carrera & 2,68 & \multirow{3}{*}{3,344} \\
\hline & $\begin{array}{l}\text { 3- Aumentar/mejorar las oportunidades } \\
\text { profesionales }\end{array}$ & 3,38 & \\
\hline & $\begin{array}{l}\text { 12- Desarrollar mis habilidades } \\
\text { profesionales }\end{array}$ & 3,97 & \\
\hline \multirow{4}{*}{$\begin{array}{l}\text { Motivos } \\
\text { Prácticos }\end{array}$} & $\begin{array}{l}\text { 7- Debido a las exigencias asociadas a mi } \\
\text { trabajo }\end{array}$ & 3,86 & \multirow{4}{*}{4,123} \\
\hline & $\begin{array}{l}\text { 8- Deseo de aumentar/desarrollar mis } \\
\text { perspectivas/ideas pedagógicas }\end{array}$ & 4,39 & \\
\hline & $\begin{array}{l}\text { 9- Alterar la forma como organizo el } \\
\text { proceso de enseñanza-aprendizaje }\end{array}$ & 3,97 & \\
\hline & $\begin{array}{l}\text { 10- Adquirir objetivos para que mi } \\
\text { enseñanza sea más eficaz }\end{array}$ & 4,28 & \\
\hline
\end{tabular}


ROGÉRIO MANUEL FERRINHO FERREIRA, SIXTO CUBO DELGADO Y VITO JOSÉ DE JESUS CARIOCA

MOTIVACIONES, NECESIDADES E IMPORTANCIA ATRIBUIDA A LA FORMACIÓN CONTINUA EN EL DESARROLLO DE HABILIDADES PROFESIONALES DEL PROFESORADO EN LA ENSEÑANZA DE ENFERMERÍA

\begin{tabular}{|c|c|c|c|}
\hline SUBCATEGORÍAS & ÍTEMS & $\begin{array}{c}\text { MEDIA } \\
\text { DE LOS } \\
\text { ÍTEMS }\end{array}$ & $\begin{array}{l}\text { MEDIA DE LOS } \\
\text { ÍTEMS DE LA } \\
\text { SUBCATEGORÍA }\end{array}$ \\
\hline \multirow{5}{*}{$\begin{array}{l}\text { Motivos } \\
\text { Emancipadores }\end{array}$} & 2- El placer asociado al estudio & 4,13 & \multirow{5}{*}{4,160} \\
\hline & 4- Promover mi desarrollo personal & 4,56 & \\
\hline & $\begin{array}{l}\text { 5- Desarrollar nuevas ideas/propuestas para } \\
\text { mi trabajo/educación }\end{array}$ & 4,48 & \\
\hline & 6- Aumentar mi autoestima & 3,39 & \\
\hline & 11- Saber más vale siempre la pena & 4,23 & \\
\hline \multirow{2}{*}{$\begin{array}{l}\text { Motivos } \\
\text { Políticos }\end{array}$} & $\begin{array}{l}\text { 15- Implementar las políticas/medidas del } \\
\text { Ministerio }\end{array}$ & 3,28 & \multirow{2}{*}{3,397} \\
\hline & $\begin{array}{l}\text { 16- Implementar las políticas/medidas de la } \\
\text { institución }\end{array}$ & 3,51 & \\
\hline \multirow{2}{*}{$\begin{array}{l}\text { Motivos } \\
\text { Pedagógicos }\end{array}$} & $\begin{array}{l}\text { 13- Realizar funciones específicas en la } \\
\text { escuela }\end{array}$ & 3,35 & \multirow{2}{*}{3,726} \\
\hline & $\begin{array}{l}\text { 14- Compartir ideas y experiencias con los } \\
\text { compañeros }\end{array}$ & 4,10 & \\
\hline
\end{tabular}

Los ítems que se destacaron, con medias superiores a las medias de todos los ítems $(3,848)$, fueron los siguientes:

- Ítem 2: El placer asociado al estudio (4,13);

- Ítem 4: Promover mi desarrollo personal $(4,56)$;

- Ítem 5: Desarrollar nuevas ideas/propuestas para mi trabajo/educación $(4,48)$;

- Ítem 7: Debido a las exigencias asociadas a mi trabajo $(3,86)$;

- Ítem 8: Deseo de aumentar/desarrollar mis perspectivas/ideas pedagógicas $(4,39)$;

- Ítem 9: Alterar la forma como organizo el proceso de enseñanza-aprendizaje $(3,97)$;

- Ítem 10: Adquirir objetivos para que mi enseñanza sea más eficaz $(4,28)$;

- Ítem 11: Saber más vale siempre la pena (4,23);

- Ítem 12: Desarrollar mis habilidades profesionales $(3,97)$;

- Ítem 14: Compartir ideas y experiencias con los compañeros $(4,10)$.

\subsection{Necesidades actuales de formación continua}

En la categoría "Necesidades actuales de formación continua" la media fue de 3,76 , con una desviación típica de 0,727 . Los ítems que presentan medias superiores a la media global de esta categoría son los siguientes, como se puede observar en el Cuadro 3: 
- Utilizar las tecnologías de información y comunicación $(3,88)$,

- Evaluar $(3,78)$ y

- Investigar $(4,26)$.

La necesidad menos valorada por estos profesores fue la siguiente: «Identificarse con la institución", con una media de 3,40. La desviación típica es superior a la desviación típica global en esta categoría $(0,727)$ en todos los ítems. Es importante destacar el hecho de que los ítems presentan un coeficiente de variación muy elevado.

\section{CUADRO 3}

Medias, desviación típica y Coeficientes de Variación de los ítems de la Categoría Necesidades actuales de formación continua

\begin{tabular}{|c|c|c|c|}
\hline CATEgoría & ÍTEMS & MEDIA & $\begin{array}{l}\text { DESVIACIÓN } \\
\text { TÍPICA }\end{array}$ \\
\hline \multirow{10}{*}{$\begin{array}{l}\text { Necesidades } \\
\text { actuales de } \\
\text { formación } \\
\text { continua }\end{array}$} & $\begin{array}{l}\text { Planificar el proceso de enseñanza- } \\
\text { aprendizaje }\end{array}$ & 3,61 & 0,968 \\
\hline & $\begin{array}{l}\text { Preparar los contenidos de las unidades } \\
\text { temáticas }\end{array}$ & 3,74 & 0,894 \\
\hline & $\begin{array}{l}\text { Dar clases de forma comprensible y bien } \\
\text { organizadas }\end{array}$ & 3,72 & 0,927 \\
\hline & $\begin{array}{l}\text { Utilizar las tecnologías de información y } \\
\text { de comunicación }\end{array}$ & 3,88 & 0,916 \\
\hline & $\begin{array}{l}\text { Definir la metodología y/u organizar las } \\
\text { actividades }\end{array}$ & 3,76 & 0,868 \\
\hline & Relacionarse con los estudiantes & 3,72 & 1,002 \\
\hline & Garantizar las tutorías & 3,74 & 0,958 \\
\hline & Evaluar & 3,78 & 0,974 \\
\hline & Investigar & 4,26 & 0,879 \\
\hline & Identificarse con la institución & 3,40 & 1,064 \\
\hline
\end{tabular}

\subsection{Importancia atribuida a la formación continua en el desarrollo de las} habilidades profesionales del profesor de Educación Superior

Verificamos que en la variable «Importancia atribuida a la formación continua en el desarrollo de las habilidades profesionales del profesor de Educación Superior» la media de los ítems fue de 3,86, con una desviación típica de 0,633.

A través del Cuadro 4, podemos verificar que las categorías que presentan una media superior a la media de los ítems de la escala $(3,86)$ son: 
ROGÉRIO MANUEL FERRINHO FERREIRA, SIXTO CUBO DELGADO Y VITO JOSÉ DE JESUS CARIOCA

MOTIVACIONES, NECESIDADES E IMPORTANCIA ATRIBUIDA A LA FORMACIÓN CONTINUA EN

EL DESARROLLO DE HABILIDADES PROFESIONALES DEL PROFESORADO EN LA ENSEÑANZA DE ENFERMERÍA

- Planificar el proceso de enseñanza-aprendizaje $(4,01) ;$

- Preparar los contenidos de las unidades temáticas $(4,06)$;

- Dar clases de forma comprensible y bien organizadas (3,93);

- Utilizar las tecnologías de información y de comunicación $(4,02)$;

- Definir la metodología y/u organizar las actividades $(4,00)$;

- Relacionarse con los estudiantes (4,03);

- Garantizar las tutorías $(4,04)$ y

- Evaluar (4,03).

\section{CUADRO 4}

Media de las Categorías en la variable Importancia atribuida a la formación continua en el desarrollo de las habilidades profesionales del profesor de Educación Superior

\begin{tabular}{|l|c|c|}
\hline \multicolumn{1}{|c|}{ CATEGORÍAs } & MEDIA & DESVIACIÓN TÍPICA \\
\hline Planificar el proceso de enseñanza-aprendizaje & 4,01 & 0,662 \\
\hline Preparar los contenidos de las unidades temáticas & 4,06 & 0,686 \\
\hline Dar clases de forma comprensible y bien organizadas & 3,93 & 0,726 \\
\hline Utilizar las tecnologías de información y de comunicación & 4,02 & 0,647 \\
\hline Definir la metodología y/u organizar las actividades & 4,00 & 0,723 \\
\hline Relacionarse con los estudiantes & 4,03 & 0,774 \\
\hline Garantizar las tutorías & 4,04 & 0,729 \\
\hline Evaluar & 4,03 & 0,687 \\
\hline Investigar & 3,66 & 0,861 \\
\hline Identificarse con la institución & 3,83 & 0,769 \\
\hline
\end{tabular}

En la categoría «Planificar el proceso de enseñanza-aprendizaje», el ítem 64, «Estructura sistemáticamente sus proyectos formativos con objetivos, contenidos, metodología y evaluación», obtiene la media más elevada, de 4,13, seguido del ítem 11, «Selecciona un conjunto de procedimientos metodológicos, que sean adecuados a la forma que están expuestos los contenidos temáticos y que se ciñan a los mismos", con 4,12.

En la categoría "Preparar los contenidos de las unidades temáticas», el ítem 22, "Selecciona contenidos, que contribuyan para una formación actualizada", obtiene la media más elevada de esta categoría con 4,19, lo que muestra la importancia que atribuyen a la formación continua. A continuación tenemos el ítem 42, «Organiza los contenidos, de manera que sean accesibles a los estudiantes y favorezcan su aprendizaje», con una media de 4,16. 
En relación a la categoría «Dar clases de forma comprensible y bien organizadas", la media más elevada $(4,09)$ se obtuvo en el ítem 3, «Explica los contenidos a los estudiantes de una forma comprensible y organizada, ajustando la enseñanza en función del feedback recibido (por la expresión facial, gestos, preguntas, etc.)».

En la categoría "Utilizar las tecnologías de información y de comunicación», fue más valorizado el ítem 68 , «Construye materiales de apoyo docente usando los medios informáticos», con una media de 4,10.

En relación a "Definir la metodología y/u organizar las actividades", el ítem con una media más elevada es el ítem 45, "Reconoce la importancia de diversificar la utilización de metodologías docentes" con una media de 4,23.

En la categoría "Relacionarse con los estudiantes» merece destacarse el ítem 62, "Estimula a los estudiantes a alcanzar los objetivos de aprendizaje", con una media de 4,21 (Cuadro 8).

El ítem que tiene la media más elevada en la categoría "Garantizar las tutorías" es el ítem 7, "Acompaña y orienta a los estudiantes durante el proceso de formación", con una media de 4,22. A continuación tenemos el ítem 47, "Demuestra actitudes de escucha activa y de empatía, en su papel de tutor/orientador», con la media de 4,19.

En relación a la categoría «Evaluar», el ítem 8 , «Evalúa con rigor y toma de decisiones sobre el nivel de aprendizaje alcanzado por los estudiantes", tiene la media más elevada con 4,11, seguido del ítem 56, «Utiliza una técnica de evaluación, centrada en el aprendizaje del estudianten, con una media de 4,10.

En la categoría "Investigar", sobresale el ítem 9, "Participa en proyectos de investigación de la/s área/s científica/s de las que forma parten, con una media de 4,01, seguido por el ítem 19, «Muestra una actitud interrogante en relación al desarrollo de proyectos de investigación que se basan en cuestiones relativas a la docencia», con una media de 3,99.

Relativamente a la importancia atribuida a la formación continua en el desarrollo de las habilidades profesionales en relación a la categoría "Identificarse con la institución", constatamos que el ítem 40, «Asume la responsabilidad de sus ideas $\mathrm{y}$ de las posiciones que adopta", presenta la media más elevada $(4,15)$.

\section{Discusión}

Asumimos que la motivación es el motor del aprendizaje y de los procesos de formación y desarrollo profesional del profesor. Durante la aplicación del cuestionario, identificamos como principales motivaciones de los profesores para la participación en acciones de formación y/o de desarrollo profesional continuo los motivos prácticos (que engloban la resolución de problemáticas y de exigencias relacionadas con la docencia) y los motivos emancipadores (asociados al propio desarrollo del profesor), en detrimento de los motivos políticos. Estos hallazgos son compatibles con los resultados obtenidos en los estudios de Correia (2012), Correia y Flores (2009), Flores et al. (2009) y Forte (2009). 
Para estos profesores del área de Enfermería de instituciones públicas portuguesas, los motivos más valorados son la promoción del desarrollo personal, el desarrollo de nuevas ideas/propuestas para mi trabajo/educación, el deseo de aumentar/desarrollar mis perspectivas/ideas pedagógicas, la adquisición de objetivos para que mi enseñanza sea más eficaz, el saber más vale siempre la pena, el placer asociado al estudio y el desarrollo de mis habilidades profesionales. Debemos destacar que la progresión en la carrera profesional es el motivo menos valorizado por estos profesores.

Cuando analizamos los resultados del cuestionario en relación a «identificación de las necesidades de formación continua de los profesores, procurando mejorar su desempeño profesional», destacan las necesidades relacionadas con la investigación, la utilización de las tecnologías de información y comunicación, así como las relacionadas con las necesidades de evaluación (con medias superiores a la media global de esta categoría). Estas necesidades manifiestan la preocupación-deseo de estos profesores en relación con el desarrollo de ciertas áreas del «saber», del «saber hacer» y del "saber ser», con la finalidad de poder asumir de forma notable los roles de facilitadores del aprendizaje y de la motivación de los estudiantes. Roles éstos que deben estar contemplados dentro de los supuestos que guían la intervención del profesor y del estudiante de la Educación Superior.

Observamos que las necesidades relacionadas con la evaluación y la investigación adquieren, también, un mayor relieve. Estas necesidades corresponden a las actividades realizadas por el profesor, así como a las diferentes áreas de formación con un importante peso institucional. La evaluación es una competencia del profesor y conlleva grandes repercusiones en los estudiantes, evaluación entendida como un concepto que implica la participación activa de los estudiantes, de forma que tenga un papel importante en la mejora de su aprendizaje.

De acuerdo con el paradigma de aprendizaje, estos procesos deberán ser más amplios y diversificados, de forma que permitan una respuesta que se ajuste a la complejidad y subjetividad de cada contexto de aprendizaje (Borralho, 2012). Investigar es una de las competencias del profesor de Educación Superior y es, igualmente, una de las mayores exigencias de las instituciones de Educación Superior (Mas, 2016). Las necesidades de formación en investigación se basan en la convicción de que los profesores son fuente de conocimientos, poseedores de saberes específicos ligados a su área científica de enseñanza, por lo que de forma inherente son privilegiados ya que pueden realizar investigaciones sobre el área que enseñan, así como sobre varias cuestiones relacionadas con la Educación Superior.

Las necesidades relacionadas con la identificación con la institución fueron las menos valorizadas, conjuntamente con la necesidad de utilizar las tecnologías de información y comunicación.

Cuando analizamos la importancia del desarrollo profesional y, dentro del mismo, la importancia de la formación continua para la consolidación del profesionalismo docente a través del aprendizaje que está implícito en la adquisición de competencias profesionales, que permiten al profesor aprender, intervenir y 
ROGÉRIO MANUEL FERRINHO FERREIRA, SIXTO CUBO DELGADO Y VITO JOSÉ DE JESUS CARIOCA MOTIVACIONES, NECESIDADES E IMPORTANCIA ATRIBUIDA A LA FORMACIÓN CONTINUA EN EL DESARROLLO DE HABILIDADES PROFESIONALES DEL PROFESORADO EN LA ENSEÑANZA DE ENFERMERÍA

transformar la práctica, como defienden algunos autores (Almeida, 2012; Cadório y Veiga Simão, 2013; Correia, 2012; Correia y Flores, 2009; Eirín Nemiña et al., 2009; Esteves, 2009; Ferreira et al., 2012; Forte, 2009; Flores et al., 2009; Lee, 2011; Marcelo, 2009; Mas, 2016; Murta, 2013; Ricardo, 2010), constatamos en la variable «identificación de la importancia atribuida a la formación continua en el desarrollo de las habilidades profesionales del profesor» que estos profesores atribuyen mayor importancia a las siguientes categorías dentro de la formación continua para el desarrollo de la habilidades profesionales: «Preparar los contenidos de las unidades temáticas», "Garantizar las tutorías», "Relacionarse con los estudiantes», «Evaluar», "Utilizar las tecnologías de información y de comunicación", "Planificar el proceso de enseñanza-aprendizaje», "Definir la metodología y/u organizar las actividades»y "Dar clases de forma comprensible y bien organizadas".

La competencia de preparar los contenidos de las unidades temáticas, cuya relevancia desde el punto de vista didáctico es reconocida (Zabalza, 2010), fue la categoría más valorada $(4,06)$ por estos docentes. Esta competencia profesional, que tiene una dimensión didáctica del tratamiento de los contenidos, teniendo en cuenta la selección, la secuencia y la estructura didáctica de los mismos, es asumida como determinante para la calidad de la enseñanza y del aprendizaje que los estudiantes realizan. Estos datos refuerzan la idea de que estos profesores del área científica de Enfermería están en sintonía con los retos derivados del proceso Bolonia, concretamente en relación a la manera de enfrentar el papel de la formación y del proceso enseñanza-aprendizaje.

La importancia atribuida a la formación continua en el desarrollo de las habilidades profesionales en la categoría de "Garantizar las tutorías» (con una media de 4,04), según estos profesores, está en consonancia con lo que preconiza Zabalza (2010), de que el profesor debe formarse para poder garantizar adecuadamente las tutorías. Las instituciones deben tomar algunas medidas para mejorar la formación de los profesores en este tipo de habilidades (Rodríguez-Hoyos, Calvo Salvador y Haya Salmón, 2015). Para poder apoyar a los estudiantes y ayudarlos, en la solución de los problemas asociados a su aprendizaje, el profesor de Educación Superior debe poseer un conjunto de características y dominio de técnicas que le permitan garantizar esta función con calidad. En el estudio de Sim-Sim, Marques, Frade y Chora (2013), donde participaron profesores y estudiantes del área de Enfermería, se verificó que los estudiantes identificaron dos aspectos en el papel del tutor: el aspecto pedagógico, dirigido a la orientación del profesor como facilitador de la progresión del estudiante de cara a la profesión que irá a seguir, y el aspecto relacional, que se encuentra asociado a las interacciones que están implícitas en la relación del profesor con los estudiantes en las tutorías. A este propósito, Sim-Sim et al. (2013) consideran que el aspecto pedagógico no está muy desarrollo dentro de la formación de estos profesores de enfermería, por lo que sugiere el desarrollo de actividades en el campo pedagógico como forma de asegurar una orientación tutorial más eficaz. Por el contrario en el aspecto relacional la formación se encuentra más desarrollado. Las competencias de "Relacionarse con los estudiantes» $\mathrm{y}$ «Evaluar» referentes a este aspecto del estudio tienen una media de 4,03. 
En relación con la competencia de "Relacionarse con los estudiantes", estos resultados suponen un reconocimiento por medio de la formación desarrollada durante el proceso formativo de los profesores y de la importancia dada al desarrollo de las interacciones entre los diferentes intervinientes. La forma en la que el profesor interacciona con los estudiantes, dentro del proceso formativo, puede provocar cambios a nivel de conocimientos, de comportamientos y de sentimientos (Zabalza, 2010; Tabera Galván, Álvarez Comino, Hernando Jerez y Rubio Alonso, 2015). En el estudio realizado por Kai Yung, Heng y Jiang (2009), estudiantes universitarios chinos, destacaron a los profesores que demostraban preocupación, paciencia, que los incentivaban, que eran responsables y que sobresalían en la relación con los estudiantes. Los estudiantes se sentían más seguros cuando los profesores les ofrecían ayuda y expresaban su preocupación por ellos. Debemos evidenciar todavía a los profesores que muestran una actitud más democrática, más simpática y sincera con los estudiantes.

Estos profesores consideran importante la formación en el desarrollo de la competencia de "evaluar", ya que consideran esta competencia como un mecanismo crucial para, así, poder comprobar si los estudiantes poseen las facultades necesarias para el ejercicio de la profesión que ambicionan tener (Zabalza, 2010). La importancia atribuida por estos profesores a la formación continua en el desarrollo de esta competencia es fundamental ante los cambios en las prácticas de evaluación de la Educación Superior, ya que constantemente surgen nuevos métodos de evaluación que procuran impulsar y regularizar los procesos de estudio y de aprendizaje, siendo estos nuevos métodos más compatibles con una evaluación formativa, tal como se manifiesta en los resultados del estudio de Bidarra, Barreira, Vaz-Rebelo, Monteiro y Alferes (2013), referente a la percepción de los estudiantes frente a la evaluación de la enseñanza en la Educación Superior. El profesor de Enseñanza Superior, a la luz del paradigma de aprendizaje, debe fomentar una evaluación de naturaleza formativa y ésta debe ser considerada como parte integrante de los procesos de enseñanza-aprendizaje, para contribuir a la mejoría del aprendizaje de los estudiantes (Borralho, 2012).

La categoría «Utilizar las tecnologías de información y de comunicación» tiene una media de 4,02. La valorización dada a esta competencia por los profesores es coherente con su importancia dentro de las actividades desarrolladas por el profesor de Educación Superior, sobre todo, si tenemos en cuenta que las nuevas tecnologías de información provocaron profundas alteraciones en el papel desempeñado por el profesor y en los métodos de enseñanza-aprendizaje. Además, la incorporación de nuevas tecnologías facilita el autoaprendizaje de los estudiantes y permite que el profesor asuma su papel orientador con la finalidad de facilitar el aprendizaje de los mismos (Borralho, 2012; Villalta Paúcar, Guzmán y Nussbaum, 2015).

La importancia atribuida por estos profesores a la formación continua en el desarrollo de la competencia de "Planificar el proceso de enseñanza-aprendizaje» (con una media de 4,01) se encuentra en concordancia con lo preconizado por 
ROGÉRIO MANUEL FERRINHO FERREIRA, SIXTO CUBO DELGADO Y VITO JOSÉ DE JESUS CARIOCA MOTIVACIONES, NECESIDADES E IMPORTANCIA ATRIBUIDA A LA FORMACIÓN CONTINUA EN EL DESARROLLO DE HABILIDADES PROFESIONALES DEL PROFESORADO EN LA ENSEÑANZA DE ENFERMERÍA

Veiga Simão et al. (2009), que sostienen que la formación continua es considerada como un elemento de referencia en la planificación de los procesos de enseñanza-aprendizaje. El papel de la formación continua puede ser determinante para la modernización de los profesores, para cuestionar las prácticas utilizadas y para el desarrollo de nuevos métodos de planificación del proceso de enseñanza-aprendizaje, que deben estar ajustados al paradigma de aprendizaje (Veiga Simão et al., 2009). En relación con esta competencia se concluye que el profesor, al planificar el proceso de enseñanza-aprendizaje, debe poner al estudiante como eje central en el proceso de aprendizaje, fomentando la aplicación de modelos centrados en la comprensión y en el desarrollo de capacidades (Borralho, 2012).

La importancia atribuida a la formación continua en el desarrollo de la competencia de definir la metodología y/u organizar las actividades (con una media de 4,00) se ajusta al paradigma del aprendizaje, que supone la utilización de metodologías y el uso de actividades que promuevan el aprendizaje del estudiante, mediante la promoción de metodologías participativas y cooperativas que fomenten la autonomía del estudiante (Borralho, 2012; Gonçalves, 2012; Villalta Paúcar et al., 2015).

En el análisis de la formación continua en el desarrollo de la competencia de "Dar clases de forma comprensible y bien organizadas» (con una media de 3,93) se destaca la necesidad del profesor de conciliar las necesidades de aprendizaje de los estudiantes con la comprensión de la información transmitida. En el estudio realizado por Gonçalves (2012), se verificó que, en relación con las representaciones que los docentes tienen sobre las funciones que el profesor de enfermería desarrolla, se da especial importancia a la transmisión de materia curricular y a los conocimientos del propio profesor.

La poca importancia atribuida, por estos profesores, a la formación continua para el desarrollo de la competencia investigar (con una media de 3,66) no se ajusta a los tiempos actuales ni a las exigencias de acreditación de los cursos de Educación Superior (Mas, 2016). Estos resultados generan alguna aprensión ya que es muy importante la investigación a nivel de enfermería, debido a que permite el desarrollo de conocimientos, así como la afirmación de la enfermería como disciplina científica. También se debe resaltar la importancia que para el área de enfermería supone que los profesores de dicha área tengan producción científica.

Es evidente la incoherencia existente entre destacar la investigación como la necesidad actual de formación más valorizada (con una media de 4,26) dentro de las categorías presentadas y, por otro lado, ser la categoría menos valorizada (con una media de 3,66), de entre las competencias profesionales utilizadas para medir la dimensión clave de este estudio, en lo referente a la importancia atribuida por los profesores de Educación Superior a la formación continua en el desarrollo de las habilidades profesionales.

Estos resultados nos permiten vislumbrar algunas implicaciones para la práctica de la formación continua en la Educación Superior: 
ROGÉRIO MANUEL FERRINHO FERREIRA, SIXTO CUBO DELGADO Y VITO JOSÉ DE JESUS CARIOCA

MOTIVACIONES, NECESIDADES E IMPORTANCIA ATRIBUIDA A LA FORMACIÓN CONTINUA EN

EL DESARROLLO DE HABILIDADES PROFESIONALES DEL PROFESORADO EN LA ENSEÑANZA DE ENFERMERÍA

1. Las instituciones resaltan la importancia de las motivaciones de los profesores de Educación Superior para participar en acciones de formación y/o desarrollo profesional continuo en detrimento de sus motivaciones en los procesos formativos y de aprendizaje.

2. Las instituciones de Educación Superior deben adquirir mecanismos que permitan la identificación de las necesidades de formación de los profesores, con la finalidad de construir proyectos de formación que fomenten el aprendizaje del profesor dentro de su trabajo (Veiga Simão et al., 2009) y el desarrollo de sus habilidades profesionales.

3. Los planes de formación deben conciliar las necesidades e intereses estratégicos de las instituciones de Educación Superior con las necesidades de formación de los profesores (García Sanz y Maquilón Sánchez, 2010; Veiga Simão et al., 2009), teniendo en cuenta que la formación debe responder a las necesidades de los ejes centrales de la escuelas, que son los estudiantes y los profesores (Lee, 2011).

4. La formación continua debe englobar áreas propias de las competencias del profesor, de acuerdo con las necesidades identificadas y con la importancia que es atribuida por los mismos, de forma que contribuya a su desarrollo profesional y a que el profesor asuma su papel de facilitador de la enseñanza, enseñanza ésta que se centra en el aprendizaje de los estudiantes. Al asumir el papel central y la importancia que la formación continua posee en el desarrollo de las habilidades del profesor (Ricardo, 2010), concluimos que la formación frente a un contexto de trabajo institucional específico debe conseguir responder a las siguientes áreas:

- Planificar el proceso de enseñanza-aprendizaje;

- Preparar los contenidos de las unidades temáticas;

- Dar clase de forma comprensible y bien organizada;

- Utilizar las tecnologías de información y de comunicación;

- Definir la metodología y/u organizar las actividades;

- Relacionarse con los estudiantes;

- Garantizar las tutorías;

- Evaluar;

- Investigar;

- Identificarse con la institución.

\section{REFERENCIAS BIBLIOGRÁFICAS}

Almeida, M. (2012). Desenvolvimento profissional dos docentes do ensino superior. Contributos para a compreensão do desenvolvimento profissional dos docentes que atuam na formação inicial de professores. Tese de Doutoramento não publicada. Universidade de Lisboa, Lisboa.

Bidarra, M.; Barreira, C.; Vaz-Rebelo, M.; Monteiro, F. y Alferes, V. (2013). Perceptions of students of learning evaluation in higher education preliminary analysis of the 
94 ROGÉRIO MANUEL FERRINHO FERREIRA, SIXTO CUBO DELGADO Y VITO JOSÉ DE JESUS CARIOCA MOTIVACIONES, NECESIDADES E IMPORTANCIA ATRIBUIDA A LA FORMACIÓN CONTINUA EN

EL DESARROLLO DE HABILIDADES PROFESIONALES DEL PROFESORADO EN LA ENSEÑANZA DE ENFERMERÍA

questionnaire replies AVENA. En M. E. Chaleta (Coord.). Atas da II International Conference "Learning and Teaching in Higher Education" and "Learning Orchestration in Higher Education» (pp. 147-155). Évora: Universidade de Évora. Recuperado de http:// dspace.uevora.pt/rdpc/bitstream/10174/9733/1/LTHE_2013_ACTAS.pdf.

Borralho, A. (2012). As aprendizagens e as Práticas de Ensino e de Avaliação. Um eixo crucial para a qualidade no ensino superior. CIEP - Comunicações - Em Congressos Científicos Nacionais. Évora: Universidade de Évora. Recuperado de http://dspace. uevora.pt/rdpc/bitstream/10174/8221/3/Aprendizagem_no_ensino_superior-relacoes_ com_a_pratica_docente.pdf.

Cadório, I. y Veiga Simão, A. (2013). Mudanças nas conceções e práticas dos professores. Lisboa: Edições Vieira da Silva, Lda.

Correia, E. (2012). Contextos e oportunidades de formação e desenvolvimento profissional. Um estudo com professores de informática. Tese de Doutoramento não publicada. Universidade do Minho, Braga.

Correia, E. y Flores, A. (2009). Experiências formativas e de desenvolvimento profissional de professores de TIC e áreas afins: alguns resultados de um estudo em curso. En Atas do X Congresso Internacional Galego-Português de Psicopedagogia. Braga: Universidade do Minho.

Cunha, M. I. (2010). Impasses contemporâneos para a pedagogia universitária no Brasil. En C. Leite (Ed.). Sentidos da pedagogia no ensino superior (pp. 63-74). Porto: CIIE/Livpsic.

Eirín Nemiña, R.; García Ruso, H. y Montero Mesa, L. (2009). Desarrollo profesional y profesionalización docente: perspectivas y problemas. Profesorado. Revista de Currículum y Formación del Profesorado, 13, 1-13. Recuperado de http://recyt.fecyt.es/index.php/ profesorado/article/view/42344/24284.

Esteves, M. (2009). Construção e desenvolvimento das competências profissionais dos professores. Sísifo. Revista de Ciências da Educação, 08, 37-48. Recuperado de http:// sísifo.fpce.ul.pt.

Fernandes, P. (2010). A Avaliação da aprendizagem no ensino superior. Possibilidades e limites de uma prática formativa/avaliativa. En C. Leite (Ed.). Sentidos da pedagogia no ensino superior. Porto: CIIE/Livpsic.

Ferreira, R.; Pereira, M. y Xavier, S. (2012). The training and development of skills in teacher. Journal of Nursing UFPE on line, 6, 2298-2306. Recuperado de http://www.revista.ufpe. $\mathrm{br} /$ revistaenfermagem/index.php/revista/article/view/2755.

Flores, M.; Veiga Simão, A.; Rajala, R. y Tornberg, A. (2009). Possibilidades e desafios da aprendizagem em contexto de trabalho: Um estudo Internacional. En M. Flores y A. Veiga Simão (Orgs.). Aprendizagem e desenvolvimento profissional de professores: Contextos e Perspetivas. Mangualde: Edições Pedago, Lda.

Forte, A. (2009). Colaboração e Desenvolvimento Profissional de Professores: Perspetivas e Estratégias: Um estudo realizado numa EB 2,3. Tese de Doutoramento não publicada. Universidade do Minho, Braga.

Fortin, M. F.; Côté, J. y Filion, F. (2009). Fundamentos e etapas do processo de investigação. Lisboa: Lusodidacta.

García Sanz, M. y Maquilón Sánchez, J. (2010). El futuro de la formación del profesorado universitario. REIFOP, 14, 17-26. Recuperado de http://www.aufop.com/.

Gonçalves, C. A. F. N. (2012). Conbecimento profissional e profissionalidade docente em Enfermagem. O contributo do pensamento docente. Tese de Doutoramento não publicada. Universidade de Lisboa, Lisboa. 
ROGÉRIO MANUEL FERRINHO FERREIRA, SIXTO CUBO DELGADO Y VITO JOSÉ DE JESUS CARIOCA

MOTIVACIONES, NECESIDADES E IMPORTANCIA ATRIBUIDA A LA FORMACIÓN CONTINUA EN

EL DESARROLLO DE HABILIDADES PROFESIONALES DEL PROFESORADO EN LA ENSEÑANZA DE ENFERMERÍA

González Calvo, G. y Barba, J. (2014). Formación permanente y desarrollo de la identidad reflexiva del profesorado desde las perspectivas grupal e individual. Profesorado. Revista de Currículum y Formación del Profesorado, 18 (1), 397-412. http://www.ugr. es/local/recfprof/rev181COL.12.pdf.

Januário, C.; Ferro, F.; Anacleto, F. y Henrique, J. (2009). Desenvolvimento profissional: a perceção da importância da formação contínua e das necessidades de formação em professores de Educação Física. Efdeportes, 135. Recuperado de http://www.efdeportes.com/.

Jooste, K. y Jasper, M. (2010). A framework for recognition of prior learning within a postgraduate diploma of nursing management in South Africa. Journal of Nursing Management, 18, 704-714. doi:10.1111/j.1365-2834.2010.01160.x.

Kai Yung, T.; Heng, M. y Jiang, G. H. (2009). What undergraduate students in China say about their professors' teaching. Teaching In Higher Education, 14, 147-159. doi:10.1080/13562510902757179.

Lee, I. (2011). Teachers as presenters at continuing professional development seminars in the English-as-a-foreign-language context: 'I find it more convincing'. Australian Journal of Teacher Education, 36, 29-42. http://dx.doi.org/10.14221/ajte.2011v36n2.3.

Marcelo, C. (2009). Desenvolvimento profissional docente: passado e futuro. Sísifo/Revista de Ciências da Educação, 8, 7-22. Recuperado de http://sísifo.fpce.ul.pt.

Mas, O. (2016). La influencia de la experiencia en las competencias investigadoras del profesor universitario. Revista Complutense de Educación, 27 (1), 13-34. http://dx.doi. org/10.5209/rev_RCED.2016.v27.n1.44706.

Mesquita, E. (2010). Formação inicial, profissão docente e competências para a docência: a visão dos futuros professores. EDUSER: revista de educação, 2, 3-19. Recuperado de http://www.eduser.ipb.pt.

Mestrinho, M. G. (2012). Ensino de Enfermagem. Caminhos de Mudança na formação de professores. Loures: Lusociência.

Murta, L. (2013). La formación permanente y el impacto sobre el desarrollo profesional: cómo lo perciben los profesores de educación física en España y Portugal. Tesis Doctoral no publicada. Universidad de Huelva, Huelva.

Ricardo, E. C. (2010). Discussão acerca do ensino por competências: problemas e alternativas. Cad. Pesqui, 40, 610-628. Recuperado de http://www.scielo.br/pdf/cp/v40n140/ a1540140.pdf.

Rodríguez-Hoyos, C.; Calvo Salvador, A. y Haya Salmón, I. (2015). La tutoría académica en la educación superior. Una investigación a partir de entrevistas y grupos de discusión en la Universidad de Cantabria (España). Revista Complutense de Educación, 26 (2), 467-481. http://dx.doi.org/10.5209/rev_RCED.2015.v26.n2.43745.

Santos, M. M. (2013). Formação contínua de professores em contextos laborais colaborativos - seus reflexos nas conceções e práticas profissionais. Tese de Doutoramento não publicada. Universidade de Lisboa, Lisboa.

Sim-Sim, M.; Marques, M.; Frade, M. y Chora, M. (2013). Tutoria: perspetiva de estudantes e professores de enfermagem. Revista Iberoamericana de Educación Superior (RIES), 11, 45-59. Recuperado de http://ries.universia.net/index.php/ries/article/view/221.

Tabera Galván, M.; Álvarez Comino, M.; Hernando Jerez, A. y Rubio Alonso, M. (2015). Percepción de los estudiantes universitarios de Ciencias de la Salud sobre las actitudes de los docentes y su influencia en el clima de aprendizaje. Revista Complutense de Educación, 26 (2), 275-293. http://dx.doi.org/10.5209/rev_RCED.2015.v26.n2.43028. 
96 ROGÉRIO MANUEL FERRINHO FERREIRA, SIXTO CUBO DELGADO Y VITO JOSÉ DE JESUS CARIOCA MOTIVACIONES, NECESIDADES E IMPORTANCIA ATRIBUIDA A LA FORMACIÓN CONTINUA EN

EL DESARROLLO DE HABILIDADES PROFESIONALES DEL PROFESORADO EN LA ENSEÑANZA DE ENFERMERÍA

Veiga Simão, A.; Flores, M.; Morgado, J.; Forte, A. y Almeida, M. (2009). Formação de professores em contextos colaborativos: um projeto de investigação em curso. Sísifo/Revista de Ciências da Educação, 8, 61-73. Recuperado de http://sisifo.fpce.ul.pt.

Villalta Paúcar, M.; Guzmán, A. y Nussbaum, M. (2015). Procesos pedagógicos y uso de tecnología en el aula. Revista Complutense de Educación, 26 (2), 405-424. http://dx.doi. org/10.5209/rev_RCED.2015.v26.n2.43303.

Zabalza, M. (2010). Competencias docentes del profesorado universitario: calidad y desarrollo profesional. Madrid: Narcea.

Zabalza, M. (2012). La enseñaza universitaria: el escenario y sus protagonistas (3. ${ }^{a}$ ed.). Madrid: Narcea. 